\title{
Antagonistas microbianos a Fusarium spp., como agente causal de pudrición de raíces y tallo en melón
}

\section{Microbial antagonists to Fusarium spp., a causal agent of root and stem rot in melon}

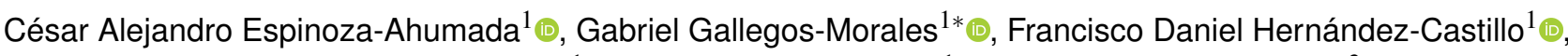 \\ Yisa María Ochoa-Fuentes ${ }^{1}$ (D), Melchor Cepeda-Siller ${ }^{1}$ (D), Francisco Castillo-Reyes ${ }^{2}$ (D) \\ ${ }^{1}$ Departamento de Parasitología, Universidad Autónoma Agraria Antonio Narro. Calzada Antonio Narro 1923, CP. 25315. Buenavista \\ Saltillo, Coahuila. México. \\ ${ }^{2}$ INIFAP, Campo Experimental, Carretera Saltillo - Zacatecas km. 342+119 \#9515, CP. 25315. Saltillo, Coahuila, México. \\ *Autor de correspondencia: ggalmor@uaaan.mx
}

Artículo científico recibido: 22 de mayo de 2018 aceptado: 18 de septiembre de 2018

RESUMEN. La pudrición de raíces y tallo en plantas de melón, ocasionado por Fusarium spp., es un factor limitante en la producción de este cultivo. El uso indiscriminado de fungicidas para el control de esta enfermedad ha causado resistencia y contaminación ambiental en estas áreas agrícolas. Una alternativa, es el uso de agentes microbianos antagónicos como Trichoderma, Bacillus y Streptomyces; los cuales pueden contribuir al manejo de la enfermedad, inducir crecimiento y mejorar la producción del cultivo. El objetivo fue evaluar la capacidad inhibitoria de 10 microorganismos antagónicos contra diferentes cepas de Fusarium spp. aisladas del cultivo de melón como agente causal de la pudrición de raíces y tallos en la región de Paila, municipio de Parras de la Fuente, Coahuila. Del área de estudio se aislaron seis cepas del género Fusarium, y con base al estudio molecular y las secuencias de nucleótidos de las regiones ITS1 e ITS4, se encontró identidad del 97 al $100 \%$ con las especies de Fusarium oxysporum y F. solani. En bioensayos in vitro, Trichoderma asperellum inhibió hasta un $74 \%$ el crecimiento de Fusarium spp. Asimismo Bacillus amyloliquefaciens un 55\% y Streptomyces mutabilis un $35 \%$. Los agentes microbianos representan un recurso para el biocontrol de la pudrición radical y del tallo del cultivo de melón.

Palabras clave: Inhibición, agentes de biocontrol, Bacillus, Trichoderma, Streptomyces.

ABSTRACT. Root and stem rot in melon plants, caused by Fusarium spp., is a limiting factor in the production of this crop. The indiscriminate use of fungicides for the control of this disease has caused resistance and environmental pollution in these agricultural areas. An alternative is the use of antagonistic microbial agents such as Trichoderma, Bacillus and Streptomyces, which can contribute to the management of the disease, induce growth and improve crop production. The objective of this study was to evaluate the inhibitory ability of 10 antagonistic microorganisms against different strains of Fusarium spp. isolated from melon cultivation as a causal agent of root and stem rot in the Paila region of the municipality of Parras de la Fuente, Coahuila. Six strains of the genus Fusarium were isolated from the study area, and based on the molecular study and nucleotide sequences of the ITS1 and ITS4 regions, 97 to $100 \%$ identity was found with the species Fusarium oxysporum and $F$. solani, respectively. In in vitro bioassays, Trichoderma asperellum inhibited the growth of Fusarium spp. by up to $74 \%$, Bacillus amyloliquefaciens by $55 \%$ and Streptomyces mutabilis by $35 \%$. Microbial agents represent a resource for the biocontrol of root and stem rot in melon cultivation.

Key words: Inhibition, biocontrol agents, Bacillus, Trichoderma, Streptomyces.

\section{INTRODUCCIÓN}

En México se reporta una producción anual de melón de 605 mil toneladas, siendo los estados de Coahuila, Michoacán y Guerrero los principales productores, con una participación del 25.2, 16.1 y $15.7 \%$ de la producción nacional (SIAP 2016). La pudrición de raíces y tallo en este cultivo ha sido un factor limitante de la producción intensiva, identificándose a Fusarium oxysporum, F. proliferatum y 


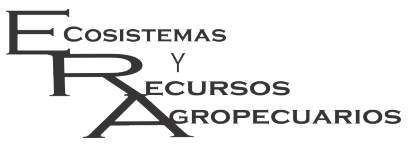

F. solani, como agentes causales de la enfermedad (Chehri et al. 2011, Zhao et al. 2014, El-Amraoui et al. 2015) y en específico a $F$. oxysporum f. sp. melonis (FOM) (Sebastiani et al. 2017). El hongo FOM es un habitante del suelo que infecta a las plantas penetrando de forma directa o por heridas de las raíces de donde se extiende hasta el xilema; provocando marchitamiento y muerte, además de persistir en el suelo debido a la producción de clamidosporas (Agrios 2005). El control genético de esta enfermedad es el método más estudiado, se han determinado los mecanismos de respuesta de defensa constitutiva o inducida de genotipos de melón a través de observaciones microscópicas, donde se observa el comportamiento de Fusarium en el xilema (Cohen et al. 2015). Para evaluar materiales genéticos por métodos moleculares van Dam et al. (2017) diseñaron 18 pares de cebadores de PCR, con base a genes efectores candidatos, que discriminan entre siete formas especiales que afectan a cucurbitáceas, lo que permite reconocer los genotipos hospederos de cada una de ellas.

Los fungicidas como benomyl, carbendazim, procloraz, fludioxonil, tebuconazole ó azoxystrobin son los más utilizados para su control, pero en contraparte impactan de forma negativa la biodiversidad del agroecosistema, con efectos nocivos en la salud humana y animal, además de que generan resistencia (Suárez et al. 2011). Se tienen registros de la resistencia a fungicidas (Brent y Hollomon 2007), lo cual no ocurre con el uso de agentes de control biológico, quienes controlan a los fitopatógenos por efecto directo, antibiosis, competencia por nutrientes y espacio, o parasitismo, o bien interfiriendo con los mecanismos patogénicos de la enfermedad y modificando la interacción patógeno-planta, al inducir resistencia adquirida local o sistémica (Bardin et al. 2015). Existe una interacción positiva de los microorganismos con plantas cultivadas, al aumentar el crecimiento, rendimiento y suprimir los efectos patológicos (Suárez et al. 2007). Por ejemplo Trichoderma spp. se reporta en tomate (Debbi et al. 2018), Bacillus spp. en melón y banano (Cao et al. 2011, Xue et al. 2015) y Streptomyces spp. en Andropogon gerardii y Lespedeza capitata (Essarioui et
Espinoza-Ahumada et al. Control Biológico de Fusarium spp. del Melón Ecosist. Recur. Agropec. 6(16):45-55,2019

al. 2017). Estas características les confieren a los agentes de control biológico una alternativa eficiente para el manejo de la enfermedad. Por lo anterior, el objetivo del presente trabajo fue identificar el agente causal de la pudrición radical del cultivo de melón de la región de Paila, Parras Coahuila y evaluar la actividad antagónica de agentes microbianos como manejo y control de esta enfermedad.

\section{MATERIALES Y MÉTODOS}

\section{Colecta de muestras de la enfermedad}

En los meses de junio y julio de 2015, se muestrearon parcelas de melón chino cantaloupe de la localidad de Paila Municipio de Parras de la Fuente, Coahuila (Tabla 1). Se colectaron plantas completas con síntomas de marchitez, necrosis en raíces y tallo, las cuales se colocaron en bolsas plásticas y trasladadas para su procesamiento al laboratorio de Fitopatología del Departamento de Parasitología, de la Universidad Autónoma Agraria Antonio Narro (UAAAN), en Buenavista, Saltillo, Coahuila, México.

\section{Aislamiento e identificación del agente causal}

Las raíces colectadas y la parte basal de las plantas se lavaron con agua corriente, se secaron a temperatura ambiente, se cortaron en pequeños trozos de tejido y se colocaron en una solución de hipoclorito de sodio al $1 \%$ durante 3 min. Después se lavaron tres veces con agua destilada estéril y se transfirieron de forma aséptica a cajas Petri con medio de cultivo papa-dextrosa-agar (PDA), acidificado con ácido láctico $(200 \mu \mathrm{L} \mathrm{L}-1)$. Las placas se incubaron a $28{ }^{\circ} \mathrm{C}$ por siete días. En función de los tipos de crecimiento y coloración se recuperaron diferentes aislamientos y transcurridos 10 días se observaron bajo microscopio compuesto las macroconidias características del género Fusarium. De los aislamientos se hizo una suspensión de conidias en agua destilada estéril para obtener esporas aisladas en placas con PDA. Transcurridas $24 \mathrm{~h}$, se transfirieron puntas de hifa en placas con PDA, hasta su desarrollo para observar crecimiento colonial. A partir de los cultivos puros, se tomaron fragmentos de la colonia y se transfirió al centro de cajas Petri con 
Tabla 1. Sitios de muestreo de Fusarium spp. en parcelas comerciales de la región productora de melón de Paila, Municipio de Parras de la Fuente, Coahuila.

\begin{tabular}{|c|c|c|c|c|}
\hline Localidad & Coordenadas & Muestras & Muestreo & Cepa \\
\hline Rancho Raúl Rivera & $\begin{array}{l}25^{\circ} 42^{\prime} 39.3^{\prime \prime} \text { LN } \\
102^{\circ} 00^{\prime} 45.5^{\prime \prime} \text { LO }\end{array}$ & $\begin{array}{l}\text { Melón, primera } \\
\text { etapa de siembra }\end{array}$ & Mayo-Junio del 2015 & FRR-1 \\
\hline Rancho La Guadalupana & $\begin{array}{l}25^{\circ} 42^{\prime} 22^{\prime \prime} \text { LN } \\
102^{\circ} 20^{\prime} 43^{\prime \prime} \text { LO }\end{array}$ & $\begin{array}{l}\text { Melón, segunda } \\
\text { etapa de siembra }\end{array}$ & Mayo-Junio del 2015 & FRG-2 \\
\hline Rancho Adolfo Flores & $\begin{array}{l}25^{\circ} 45^{\prime} 57.8^{\prime \prime} \text { LN } \\
101^{\circ} 59^{\prime} 50^{\prime \prime} \text { LO }\end{array}$ & $\begin{array}{l}\text { Melón, segunda } \\
\text { etapa de siembra }\end{array}$ & Mayo-Junio del 2015 & FAF-3 \\
\hline Rancho Elena & $\begin{array}{l}25^{\circ} 45^{\prime} 10^{\prime \prime} \text { LN } \\
102^{\circ} 24^{\prime} 47^{\prime \prime} \text { LO }\end{array}$ & $\begin{array}{l}\text { Melón, tercera } \\
\text { etapa de siembra }\end{array}$ & Mayo-Junio del 2015 & FRE-4 \\
\hline Rancho Feliciano Alarcón & $\begin{array}{l}25^{\circ} 45^{\prime} 20.2^{\prime \prime} \text { LN } \\
102^{\circ} 10^{\prime} 48.5^{\prime \prime} \text { LO }\end{array}$ & $\begin{array}{l}\text { Melón, primera } \\
\text { etapa de siembra }\end{array}$ & Mayo-Junio del 2015 & FCA -5 \\
\hline Rancho Héctor Alarcón & $\begin{array}{l}25^{\circ} 45^{\prime} 39^{\prime \prime} \text { LN } \\
102^{\circ} 10^{\prime} 53^{\prime \prime} \text { LO }\end{array}$ & $\begin{array}{l}\text { Melón, siembra de la } \\
\text { temporada pasada }\end{array}$ & Mayo-Junio del 2015 & FHA-6 \\
\hline
\end{tabular}

PDA y Synthetischer nährstoffärmer agar (SNA). Las placas inoculadas se incubaron a $28{ }^{\circ} \mathrm{C}$ por 15 días, se procedió a realizar observaciones macroscópicas de las cepas crecidas en PDA. De las cepas que se desarrollaron en medio SNA, se hicieron montajes en portaobjetos para observar las características microscópicas del hongo. Para identificar las especies, se usaron las claves taxonómicas de Leslie y Summerell (2006) mediante la observación de la morfología del micelio, fialides, microconidias y macroconidias.

\section{Identificación molecular de Fusarium}

Los aislamientos de Fusarium se sembraron en el medio de cultivo PDA y se incubaron por cinco días. La extracción del ADN se realizó mediante el método de Doyle y Doyle (1990), con modificaciones; la extracción se valoró con electroforesis en gel de agarosa al 1\%. La amplificación se realizó con el método de reacción en cadena de la polimerasa (PCR), de las regiones internas transcritas ITS1 e ITS4 entre los genes ribosomales (rDNA) 18S-5.8S y 5.8S-28S utilizando el par de iniciadores de secuencia ITS1 (TCCGTAGGTGAACCTGCGG)/ ITS4 (TCCTCCGCTTATTGATATGC). La amplificación se realizó, colocando en cada muestra buffer de enzima a $1 \mathrm{x}, 1 \mu \mathrm{L}$; dNTP's a $0.2 \mathrm{mM}, 1 \mu \mathrm{L} ; \mathrm{MgCl}$ a $2 \mathrm{mM}$, $0.4 \mu \mathrm{L}$; ITS1 a $1 \mathrm{pM}, 0.5 \mu \mathrm{L}$; ITS4 a $1 \mathrm{pM}, 0.5 \mu \mathrm{L}$; 0.5 UDO de Taq polymerasa, $0.1 \mu \mathrm{L}$; DNA problema ajustado a $50 \mathrm{ng}, 1 \mu \mathrm{L}$; y $5.5 \mu \mathrm{L}$ de agua ultrapura estéril para ajustar un volumen final de $10 \mu \mathrm{L}$. Las condiciones para el termociclador fueron: 1 ciclo de desnaturalización inicial a $94{ }^{\circ} \mathrm{C}$ por 5 min, 30 ciclos de desnaturalización a $95{ }^{\circ} \mathrm{C}$ por $10 \mathrm{~s}, 30$ ciclos de alineamiento a $57^{\circ} \mathrm{C}$ por $30 \mathrm{~s}, 30$ ciclos de extensión a $72{ }^{\circ} \mathrm{C}$ por 2 min y 1 ciclo de extensión final a 72 ${ }^{\circ} \mathrm{C}$ por 5 min (Ochoa et al, 2012). Los productos de PCR se purificaron con el kit de purificación de bandas de in vitro gen (PureLink ${ }^{\circledR}$ Quick Gel Extraction and PCR Purification Combo Kit), para proceder a su secuenciación directa en ambas direcciones, usando el algoritmo BLAST, las secuencias obtenidas fueron comparadas con las reportadas en la base de datos del banco de genes del NCBI, por sus siglas en inglés. Para la edición del árbol filogenético se hizo la alineación mediante el software Clustal Omega y el gráfico con el programa FrigTree.

\section{Pruebas de patogenicidad de Fusarium}

Se realizaron con pruebas in vitro (Sánchez et al. 1975), sumergiendo semillas de melón desinfectadas del genotipo Top Mark (altamente susceptible) en una suspensión de conidios de cada aislamiento $\left(1 \times 10^{5}\right.$ conidias por $\left.\mathrm{mL}\right)$ producidos en PDA. Se incubaron sobre placas con Agar-Agua (AA) a $28^{\circ} \mathrm{C}$, durante siete días. La severidad de la enfermedad se evaluó, mediante la escala: $0=$ sin síntomas visibles; 1 = puntos necróticos aislados en el hipocótilo; 2 = oscurecimiento en la base del hipocótilo; 3 = lesión necrótica de 1-5 mm en la base del hipocotilo; $4=$ lesión necrótica de 6-10 mm en la base del hipocótilo; 5 = lesión necrótica mayor a $11 \mathrm{~mm}$ en la base del hipocótilo. 


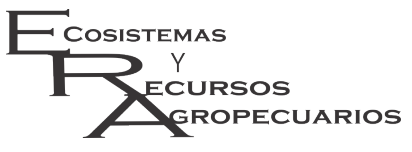

\section{Tratamientos evaluados}

Los microorganismos antagónicos evaluados fueron: Bacillus liquefaciens (BIF), B. amyloliquefaciens (BCC), B. subtillis (BSK), Trichoderma aspereIlum (TA), T. harzianum (TH2), T. viride (TV), Streptomyces longisporoflavus, (SL), S. mutabilis (SM), $S$. griseus (SG) y Streptomyces sp. (SSP), provenientes del cepario del Departamento de Parasitología de la UAAAN. El experimento se estableció bajo un diseño completamente al azar, con 78 tratamientos y cinco repeticiones. La unidad experimental consistió en una placa de Petri y la variable a medir fue el diámetro de crecimiento del hongo.

\section{Bioensayo}

La actividad inhibitoria de las 10 especies mencionadas anteriormente se evaluó por separado con seis aislamientos de Fusarium. En el caso de Trichoderma se realizó mediante la técnica de Cherif y Benhamou (1990), que consistió en depositar en un extremo de la caja Petri un explante PDA de $5 \mathrm{~mm}$ de diámetro con micelio de Fusarium de ocho días de edad y en el otro extremo de la caja se depositó un explante de $5 \mathrm{~mm}$ de las diferentes especie de Trichoderma, incubándose a $28{ }^{\circ} \mathrm{C}$ por $12 \mathrm{~h}$. Después, se midió el crecimiento radial de Fusarium cada $24 \mathrm{~h}$, para lo cual se utilizó un Vernier digital. Para medir el antagonismo de Bacillus y Streptomyces se utilizó el método de confrontación, en este caso la especie antagonista a evaluar se inoculó en los cuatro puntos cardinales de cada placa con medio de cultivo PDA y colocando al centro de la misma un explante de $5 \mathrm{~mm}$ de diámetro de Fusarium. Las placas se incubaron a $28{ }^{\circ} \mathrm{C}$, la medición de la capacidad inhibitoria se realizó con un Vernier digital, tomando el diámetro del crecimiento fúngico $(\mathrm{mm})$ en los dos puntos cardinales referentes a la inhibición. Posteriormente se determinó el porcentaje de inhibición mediante la fórmula: Porcentaje de inhibición $=\left((D C C-D C T){ }^{*} 100\right) /$ DCC, donde: $\mathrm{DCC}=$ Diámetro de la colonia control, y DCT = Diámetro de la colonia tratada.

\section{Análisis estadístico}

Se realizó la prueba Shapiro Wlik para determinar la distribución normal de los datos, un análisis
Espinoza-Ahumada et al.
Control Biológico de Fusarium spp. del Melón

Ecosist. Recur. Agropec. 6(16):45-55,2019 de varianza (ANVA) y una prueba de comparación de medias según Tukey $(p \leq 0,05)$. El análisis de los datos se realizó con el programa estadístico SAS.

\section{RESULTADOS}

\section{Aislamiento e identificación de Fusarium}

Se aislaron seis hongos, que tuvieron características típicas de Fusarium (Tabla 2). El crecimiento caracteristico de F. solani (FRR-1, FRG2 y FCA-5) mostró pigmentación blanca y beige en medio sólido PDA (Figura 1), al microscópio se observaron microconidias en fialides largas, agrupadas en falsas cabezas, de forma oval a elíptica, mono y bicelulares, las macroconidias presentaron células apicales y basales redondeadas de tres a cinco septos. F. oxysporum (FAF-3, FRE-4 y FHA-6), presentó micelio algodonoso, coloracíon café y violeta rosáceo, microscópicamente se encontraron microconidias unicelulares y bicelulares, de forma ovoide a elipsoide, en fialides cortas agrupadas en falas cabezas y macroconidias con las células apicales ligeramente curvadas, células basales truncas con tres a cinco septos.

Se corroboró la identidad de las mismas, a través de la técnica de ITS-PCR, obteniendo productos amplificados de aproximadamente $500 \mathrm{pb}$ (Figura 2), resultando los aislamientos FRR-1, FRG-2 y FCA5 como la especie Fusarium solani y los aislamientos FAF-3, FRE-4 y FHA-6 como Fusarium oxysporum, con un porcentaje de identidad del 97 al 100\% (Figura $3)$.

\section{Pruebas de patogenicidad}

Se determinó que las cepas identificadas como FRR-1, FRG-2 y FCA-5, de Fusarium solani, fueron altamente patogénicas ubicándose en la escala de severidad en el nivel 5, al igual las cepas FAF-3 y FRE-4 de Fusarium oxysporum, mientras que la cepa FHA-6 se situó en el nivel 4 y el testigo no presentó daños visibles (Figura 4).

\section{Actividad antagonista}

El porcentaje de inhibición entre microorganismos evaluados in vitro (Tabla 3 ), muestran que Tri- 
Tabla 2. Similaridad de las secuencias de nucleótidos de los aislamientos Fusarium spp. obtenidos de la región de Paila, comparadas con la base de datos del NCBI.

\begin{tabular}{ccccc}
\hline Aislado & Especie & Nucleotidos $^{x}$ & No. de acceso & IS $^{z}$ \\
\hline FRR-1 & F. solani & 421 & KU883630 & $100 \%$ \\
FRG-2 & F. solani & 477 & KU325529 & $98 \%$ \\
FAF-3 & F. oxysporum & 429 & KU097274 & $98 \%$ \\
FRE-4 & F. oxysporum & 475 & KU097274 & $97 \%$ \\
FCA-5 & F. solani & 432 & KU325529 & $99 \%$ \\
FHA-6 & F. oxysporum & 450 & KT366736.1 & $97 \%$ \\
\hline
\end{tabular}

${ }^{x}=$ Número de nucleótidos de la secuencia comparada, ${ }^{y}=$ Índice de similaridad de la secuencia comparada con las bases de datos, $\mathrm{y}^{z}=$ Número de acceso en la base de datos del NCBI.

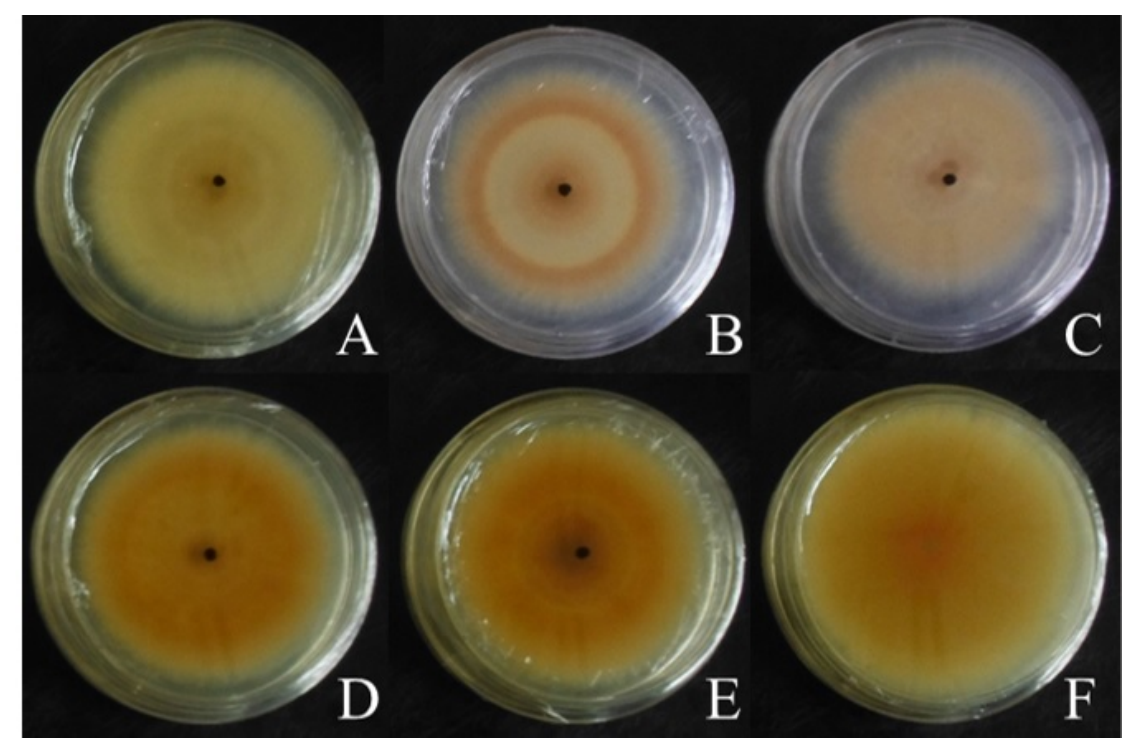

Figura 1. Crecimiento micelial de. F. solani (A, B y C) y F. oxysporum (D, E y F).

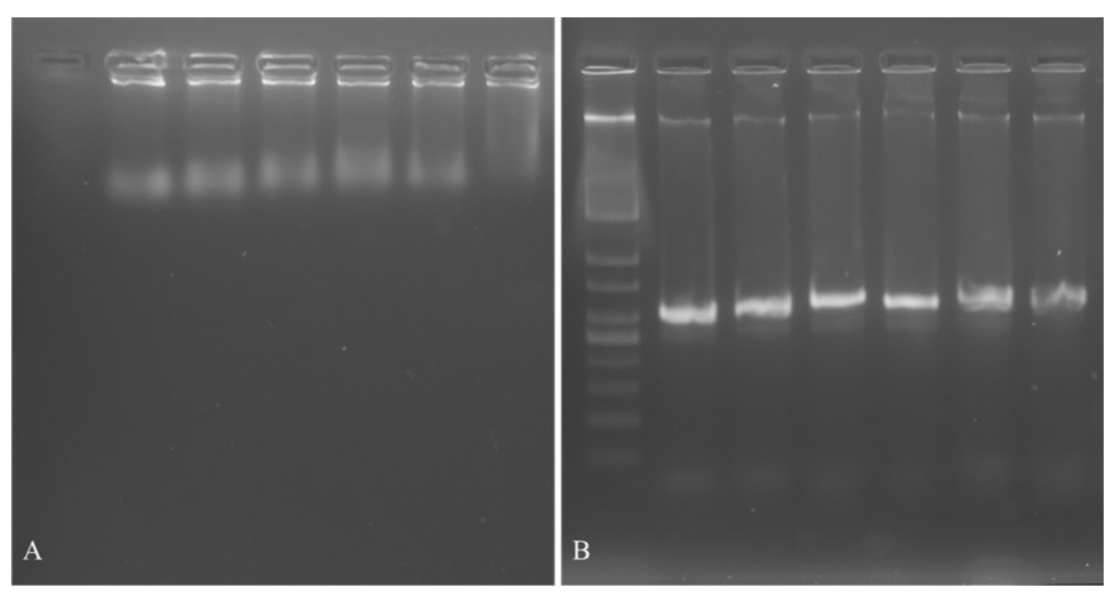

Figura 2. Gel de extracción de ADN de aislamientos de Fusarium (A). Gel de agarosa de productos de PCR obtenidos a partir de ADN de aislados de Fusarium utilizando ITS1 e ITS4 (B). 


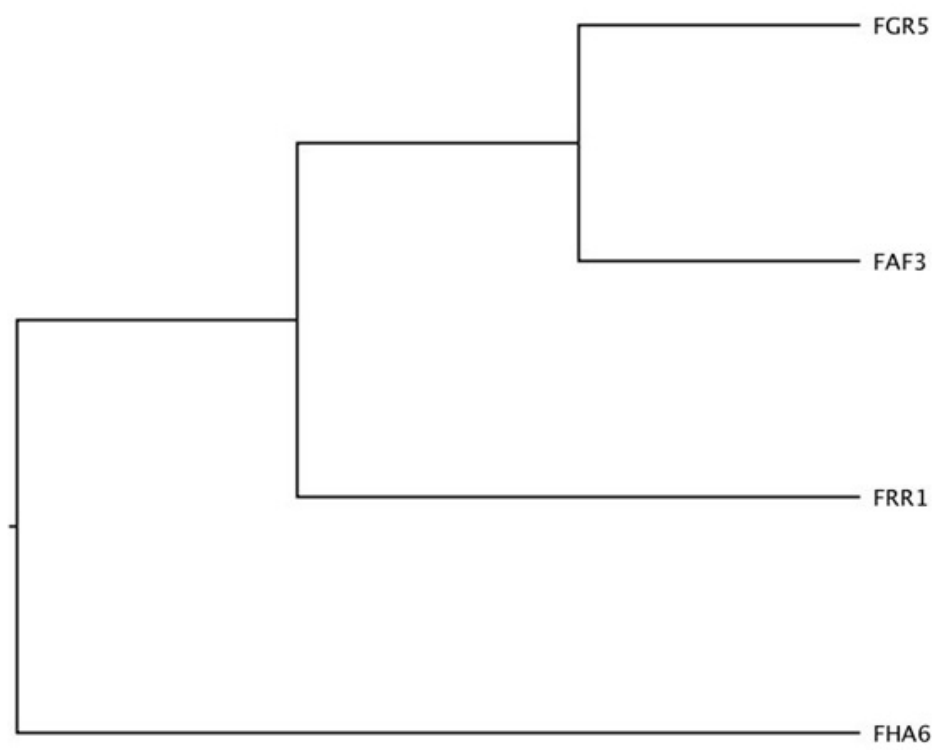

0.02

Figura 3. Dendrograma obtenido por alineación mediante Clustal Omega y FrigTree.
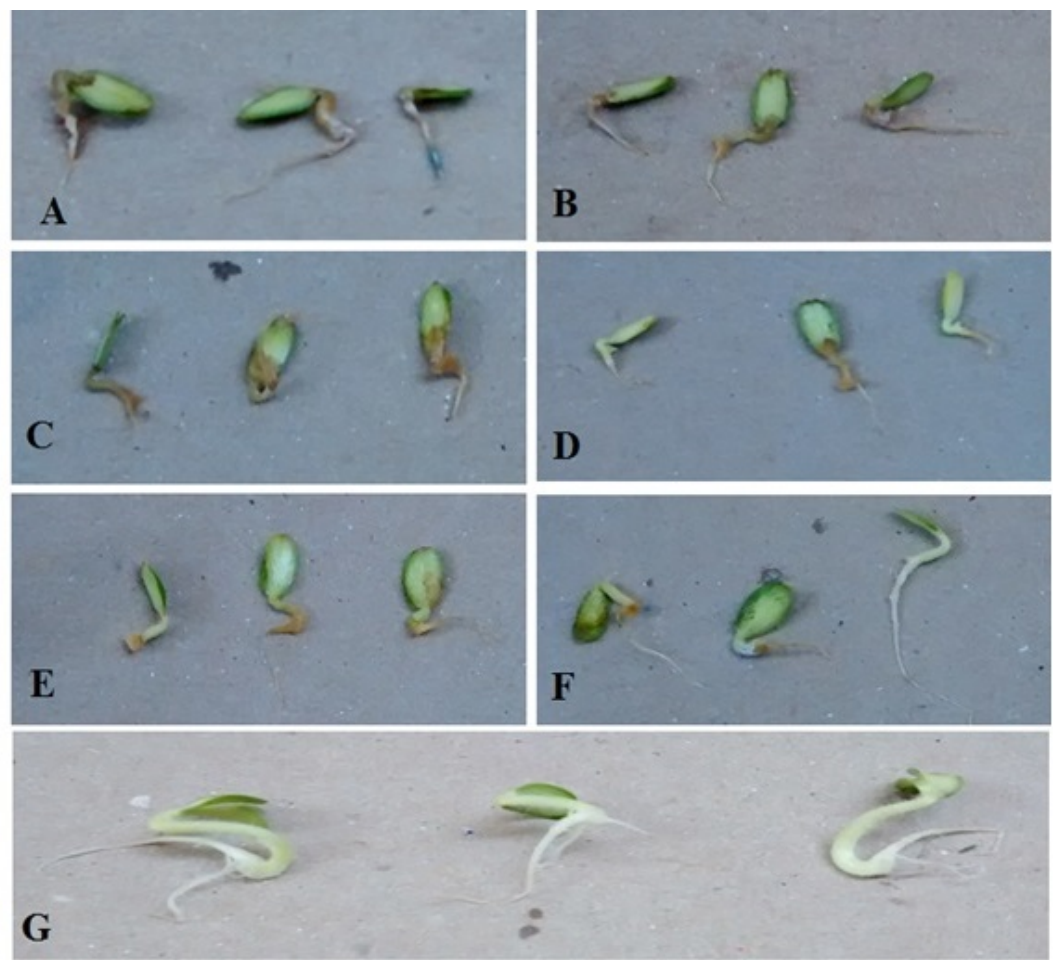

Figura 4. Pruebas de patogenicidad en semillas de melón: A) Aislamiento FRR-1; (B) Aislamiento FRG-2; (C) Aislamiento FAF-3; (D) Aislamiento FRE-4; (E) Aislamiento FCA-5; (F) Aislamiento FHA-6.; (G) Testigo sin inocular. 
Tabla 3. Porcentaje de capacidad antagónica de diferentes organismos biológicos contra cepas de Fusarium spp.

\begin{tabular}{lllllll}
\hline & \multicolumn{7}{c}{${ }^{*}$ Cepas de Fusarium spp. } \\
Organismos antagónicos & FRR-1 & FRG-2 & FAF-3 & FRE-4 & FCA-5 & FHA-6 \\
\hline B. liquefaciens & $46.9^{A, a b}$ & $38.4^{B, a b c}$ & $31.4^{B C, b c}$ & $34.8^{C D, b c}$ & $28.2^{C D, c}$ & $41.6^{D E, a b}$ \\
B. amyloliquefaciens & $51.4^{A B, a b}$ & $38.9^{B, c}$ & $37.8^{B, c}$ & $42.9^{B C, b c}$ & $41.2^{B C, b c}$ & $55.2^{C, a}$ \\
B. subtillis & $45.7^{B, a}$ & $38.6^{B, a}$ & $37.2^{B, a}$ & $42.4^{B, c a}$ & $34.6^{C, a}$ & $46.4^{D, a}$ \\
T. asperellum & $57.2^{A, b}$ & $61.5^{A, a b}$ & $64.3^{A, a b}$ & $62.3^{A, a b}$ & $54.9^{A, b}$ & $74.1^{A, a}$ \\
T. harzianum & $54.9^{A B, b}$ & $55.4^{A, b}$ & $56.1^{A, a b}$ & $60.5^{A, a b}$ & $57.9^{A, a b}$ & $64.7^{B, a}$ \\
T. viride & $49.8^{A, a b}$ & $58.3^{A, a}$ & $54.9^{A, a}$ & $51.1^{A, a b}$ & $50.3^{B C, a}$ & $64.7^{B, a}$ \\
S. mutabilis & $19.8^{C, b c}$ & $27.5^{B, a b}$ & $21.5^{C D, b c}$ & $21.0^{D, e b c}$ & $16.4^{D E, c}$ & $35.5^{E, a}$ \\
S. griseus & $3.7^{D, a}$ & $4.3^{C, a}$ & $2.4^{E, a}$ & $5.2^{F, a}$ & $4.4^{E F, a}$ & $3.4^{F, a}$ \\
S. longisporoflavus & $0.7^{D, a}$ & $0.2^{C, a}$ & $2.2^{E, a}$ & $2.0^{F, a}$ & $0.6^{F, a}$ & $0.8^{F, a}$ \\
Streptomyces sp. & $8.0^{D, b}$ & $4.8^{C, b}$ & $12.9^{D, a b}$ & $16.3^{E, a}$ & $6.2^{E F, b}$ & $5.1^{F, b}$ \\
\hline Letras mayúsculas indican comparación entre columnas, letras minúsculas indican comparación entre filas, \\
porcentajes de inhibición con diferentes letras representan diferencias ( $\mathrm{p} \leq 0.05)$.
\end{tabular}

choderma asperellum, fue el que presentó mayor efectividad biológico contra los distintos aislamientos de Fusarium aisladas de melón, alcanzando porcentaje de inhibición del 54.9 al $74.1 \%$, seguido de T. harzianum con antagonismo del 54.9 al $64.7 \%$. El aislamiento FHA-6 de $F$. oxysporum, fue el que presentó mayor susceptibilidad y FRR-1, identificada como F. solani, mostró menores porcentajes de inhibición a las diferentes especies de Trichoderma. En las evaluaciones con Bacillus spp., se encontró que $B$. amyloliquefaciens presentó los niveles más altos de control, con niveles del 37.8 al $55.2 \%$ en los aislamientos FAF-3 y FHA-6, respectivamente. Los menores porcentajes de inhibición fueron en el aislamiento FCA-5, del 28.2 y $34.6 \%$, con B. liquefaciens y $B$. subtillis, y FAF-3 con $37.8 \%$ en $B$. amyloliquefaciens (Tabla 3). Al evaluar las cuatro especies de Streptomyces como alternativa de control in vitro, se encontró bajos niveles de actividad contra las especies de este fitopatógeno. S. mutabilis alcanzó los mayores efectos antagonistas con $35.5 \%$ de inhibición contra el aislamiento FHA-6, la más susceptible, y $S$. longisporoflavus el controlador que presentó los niveles más bajos de antagonismo. La inhibición promedio más alta con respecto a los seis aislamientos de Fusarium se obtuvo que las tres especies de Trichoderma que mostraron porcentajes de inhibición del 62.4 al 54.8\%, seguido de Bacillus spp. en un rango de 44.5 al $36.9 \%$ y Streptomyces spp. de 23.6 al $1.1 \%$ (Figura 5 ).

\section{DISCUSIÓN}

Las especies F. oxysporum y F. solani son los agentes causales de la pudrición de raíces y tallos en el cultivo de melón, lo que coincide con investigaciones realizadas previamente (Chehri et al. 2011, Seo y Kin 2017). En la presente investigación, las tres especies de Trichoderma alcanzaron niveles del 49 al $74 \%$ de inhibición, valores que son similares a lo reportado por García (2015) y El-Komy et al. (2015) quienes reportan hasta el 72\% de inhibición del crecimiento de Fusarium spp. En este contexto Yanet y Cabrales (2016) en evaluaciones in vitro, encontraron que dos cepas de $T$. asperellum y una de T. longibrachiatum, confrontadas con diferentes cepas de Moniliophthora roreri, al sexto día lograron el $100 \%$ de antagonismo y concluyeron una manifestación microscópica de micoparasitismo sobre los fitopatógenos. Al respecto, Song et al. (2014) reportan que B. amyloliquefaciens inhibe un $85 \%$ el crecimiento de Fusarium, aislado del cultivo de ginseng (Panax ginseng Meyer). Se tiene evidencia que la bacteria $B$. velezensis produce tres compuestos de lipopéptidos (surfactina, iturina y fenginina), que son responsables de una fuerte actividad antagonista, contra F. oxysporum f. sp. cubense y F. oxysporum f. sp. cucumerinum (Cao et al. 2018), y además son promotoras del crecimiento vegetal, y forman esporas que son fáciles de formular y conservar hasta su inoculación (Pila 2016, Khan et al. 2017). Para el 
Inhibición de antagonistas microbianos a Fusarium spp.

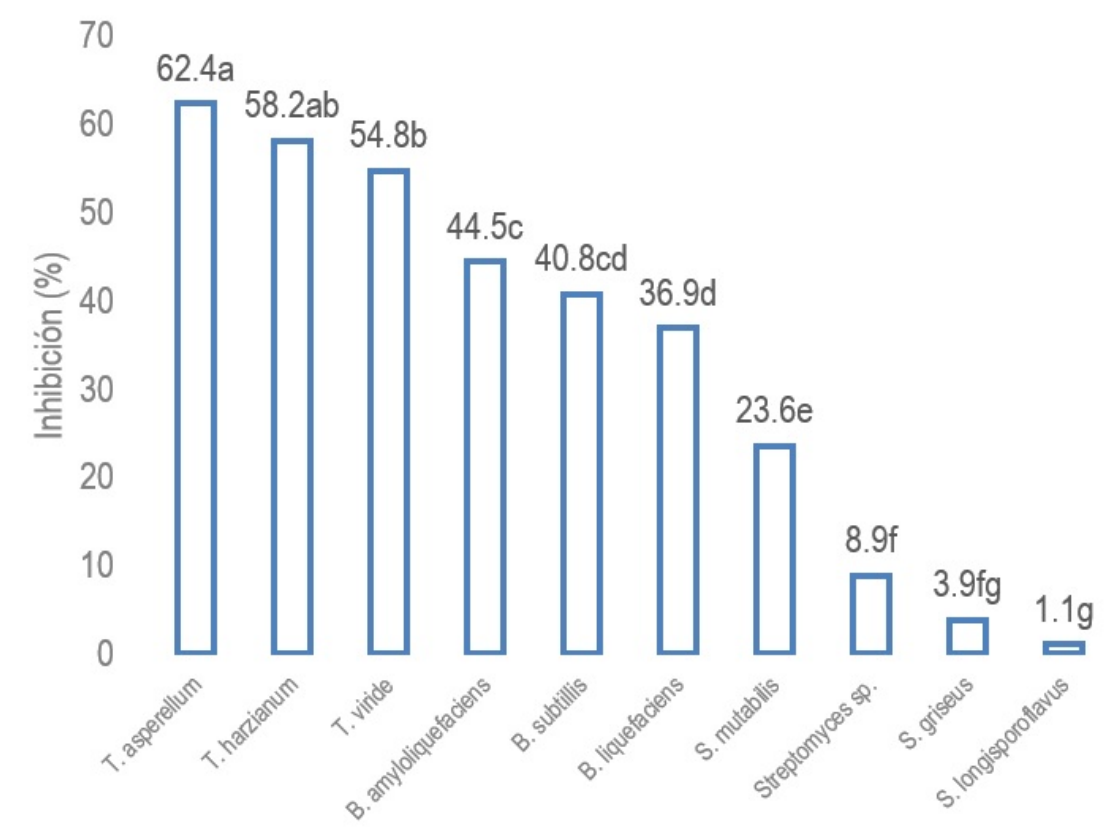

Figura 5. Antagonismo de agentes microbianos a las cepas de F. oxysporum (FAF-3, FRE-4, FHA-6) y F. solani (FRR-1, FRG-2, FCA-5).
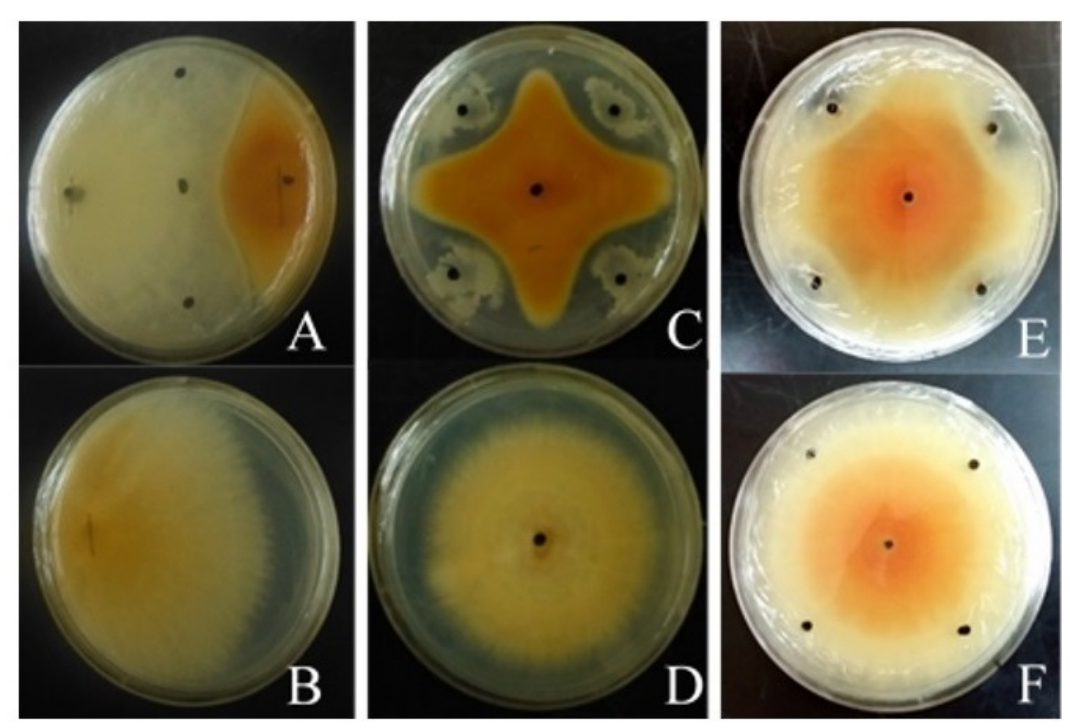

Figura 6. Trichoderma sp. vs a Fusarium spp. (A) y testigo (B); Bacillus sp. vs a Fusarium spp. (C) y testigo (D); Streptomyces sp. vs a Fusarium spp.(E) y testigo (F).

caso de S. mutabilis, Thakur et al. (2007) y Pérez et al. (2015) al evaluar Streptomyces spp. contra Fusarium encontraron bajos porcentajes de antago- nismo, lo que concuerda con la variabilidad inhibitoria que se observó en este trabajo (Figura 6). La lentitud en el desarrollo del crecimiento de las especies de 


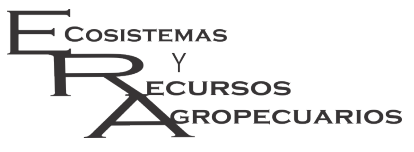

Streptomyces en medios de PDA pudiera ser el punto limitante por el que existe baja actividad de estas bacterias in vitro, ya que es común que el fitopatógeno se desarrolle más rápido que Streptomyces, de ahí la baja actividad antagónica (Dávila et al. 2013). Al respecto Kanini et al. (2013) reportan que la especie Streptomyces rochei, aislada de la rizósfera de la planta Pinus brutia, tienen potencial para inhibir el crecimiento in vitro de Fusarium oxysporum, aislado del cultivo de tomate, mientras que Martínez et al. (2017) mencionan que los actinomicetos tienen actividad inhibitoria en el crecimiento de fitopatógenos del cultivo del chile.
Espinoza-Ahumada et al. Control Biológico de Fusarium spp. del Melón Ecosist. Recur. Agropec. 6(16):45-55,2019

\section{CONCLUSIONES}

Se aisló e identificó a las especies Fusarium oxysporum y $F$. solani como agentes causales de la pudrición de raíces y tallos en el cultivo de melón. Ambas especies fueron susceptibles al antagonismo in vitro de Trichoderma asperellum y Bacillus amyIoliquefaciens, en porcentajes de capacidad inhibitoria que requieren evaluarse en condiciones de campo para el control de la enfermedad. Mientras que las especies de Streptomyces no presentaron efectividad biológica para Fusarium.

\section{AGRADECIMIENTOS}

Al CONACYT por la beca otorgada para obtener estudios de Maestría en Ciencias.

\section{LITERATURA CITADA}

Agrios GN (2005) Plant pathology. 5th Edition. Elsevier, Academic Press. California, USA. 948p

Bardin M, Ajouz S, Comby M, Lopez M, Graillot B, Siegwart M, et al. (2015) Is the efficacy of biological control against plant diseases likely to be more durable than that of chemical pesticides? Frontiers in Plant Science 6: 566. Doi: 10.3389/fpls.2015.00566.

Brent KJ, Hollomon DW (2007) Fungicide resistance: the assessment of risk. FRAC Monograph No. 1. 2nd edition. Croplife International, Brussels, Belgium. 52p

Cao Y, Pi H, Chandrangsu P, Li Y, Wang Y, Zhou H, et al. (2018) Antagonism of two plant-growth promoting Bacillus velezensis isolates against Ralstonia solanacearum and Fusarium oxysporum. Scientific Reports 8: 4360. Doi: 10.1038/s41598-018-22782-z

Cao Y, Zhang Z, Ling N, Yuan Y, Zheng X, Shen B, et al. (2011) Bacillus subtilis SQR 9 can control Fusarium wilt in cucumber by colonizing plant roots. Biology and fertility of soils 47: 495-506

Chehri K, Salleh B, Yli-Mattila T, Reddy KN, Abbasi S (2011) Molecular characterization of pathogenic Fusarium species in cucurbit plants from Kermanshah province, Iran. Saudi Journal of Biological Sciences 18: 341 351.

Cherif SS, Benhamou CS (1990) Cytochemical aspects of chitin breakdown during the parasitic action of a Trichoderma spp., on Fusarium oxysporum f. sp. radicis-lycopersici. Phytopathology. 80: 1406-1414. Doi: 10.1094/phyto-80-1406

Cohen R, Orgil G, Burger Y, Saar U, Elkabetz M, Tadmor Y, et al. (2015) Differences in the responses of melon accessions to Fusarium root and stem rot and their colonization by Fusarium oxysporum f. sp. radiciscucumerinum. Plant Pathology 64: 655-663.

Dávila MMD, Gallegos MG, Hernández CFD, Ochoa FYM, Flores OA (2013) Actinomicetos antagónicos contra hongos fitopatógenos de importancia agrícola. Revista Mexicana de Ciencias Agrícolas 4: 1187-1196. 
Debbi A, Boureghda H, Monte E, Hermosa R (2018) Distribution and genetic variability of Fusarium oxysporum associated with tomato diseases in Algeria and a biocontrol strategy with indigenous Trichoderma spp. Frontiers in Microbiology 9: 282. Doi: 10.3389/fmicb.2018.00282.

Doyle JJ, Doyle JL (1990) Isolation of plant DNA from fresh tissue. Focus 12: 13-15.

El-Amraoui B, Biard JF, Ikbal FEZ, El-Wahidi M, Kandil M, El-Amraoui M, et al. (2015) Activity of Haliscosamine against Fusarium oxysporum f. sp. melonis: in vitro and in vivo analysis. Springerplus 4: 16. Doi: 10.1186/s40064-015-0797-x

El-Komy MH, Saleh AA, Eranthodi A, Molan YY (2015) Characterization of novel Trichoderma asperellum isolates to select effective biocontrol agents against tomato Fusarium wilt. The Plant Pathology Journal 31: 50-60.

Essarioui A, LeBlanc N, Kistler HC, Kinkel LL (2017) Plant community richness mediates inhibitory interactions and resource competition between Streptomyces and Fusarium populations in the rhizosphere. Microbial Ecology 74: 157-167

Garcia L (2015) Evaluación del antagonismo de cepas de Trichoderma spp. frente a cepas de Fusarium spp. y Colletotrichum spp. aisladas de cultivos orgánicos de sábila (Aloe vera) y arándano (Vaccinium corymbosum). Revista Teckne 13: 56 - 66.

Kanini GS, Katsifas EA, Savvides AL, Karagouni AD (2013) Streptomyces rochei ACTA1551, an indigenous greek isolate studied as a potential biocontrol agent against Fusarium oxysporum f.sp. lycopersici. Biomed Research International 2013 Article D 387230. Doi: 10.1155/2013/387230

Khan N, Maymon M, Hirsch AM (2017) Combating Fusarium infection using Bacillus-based antimicrobials. Microorganisms 5: 75. Doi: 10.3390/microorganisms504007.

Leslie JF, Summerell BA (2006) The Fusarium laboratory manual. Blackwell publishing. 388p.

Martínez ZE, Aguilar EEQ, Enríquez GR (2017) Potencial biotecnológico de las actinobacterias aisladas de suelos de México como fuente natural de moléculas bioactivas: compuestos antimicrobianos y enzimas hidrolíticas. Temas de Ciencia y Tecnología 21: 39-51.

Ochoa FYM, Cerna CE, Gallegos MG, Landeros FJ, Delgado OJC, Hernández CS, et al. (2012) Identificación de especies de Fusarium en semilla de ajo en Aguascalientes, México. Revista Mexicana de Micología 36: 27-32.

Pérez RF, León J, Galindo CN (2015) Actinomicetos aislados del compost y su actividad antagonista a fitopatógenos de la papa (Solanum tuberosum spp. andigena Hawkes). Revista Mexicana de Fitopatología 33: 116-139.

Pila FES (2016) Importancia de los lipopéptidos de Bacillus subtilis en el control biológico de enfermedades en cultivos de gran valor económico. Bionatura 1: 135-138.

Sánchez LE, Endo RM, Leary JV (1975) A rapid technique for identifying the clones of Fusarium oxysporum $\mathrm{f}$. sp. lycopersici causing crown-and root-rot of tomato [Fungus diseases]. Phytopathology 65: 726-727.

Sebastiani MS, Bagnaresi P, Sestili S, Biselli C, Zechini A, Orrù, L, et al. (2017) Transcriptome analysis of the melon-Fusarium oxysporum f. sp. melonis race 1.2 pathosystem in susceptible and resistant plants. Frontiers in Plant Science 8: 362. Doi:10.3389/fpls.2017.00362.

Seo Y, Kim YH (2017) Potential reasons for prevalence of Fusarium wilt in Oriental Melon in Korea. The Plant Pathology Journal 33: 249-263. 
SIAP (2016) Servicios de Información Agroalimentaria y Pesquera. Anuario estadístico. Disponible en: https://nube.siap.gob.mx/cierreagricola/. Fecha de consulta: 8 de Agosto del 2018

Song M, Yun HY, Kim YH (2014) Antagonistic Bacillus species as a biological control of ginseng root rot caused by Fusarium cf. incarnatum. Journal of Ginseng Research 38: 136-145.

Suárez EF, Vargas GC, Lopez MJ, Capel C, Moreno J (2007) Antagonistic activity of bacteria and fungi from horticultural compost against Fusarium oxysporum f. sp. melonis. Crop Protection 26: 46-53.

Suárez MH, Hernández CFD, Gallegos MG, Lira SRH, Rodríguez HR, Aguilar CN (2011) Biocontrol of soil fungi in tomato with microencapsulates containing Bacillus subtilis. American Journal of Agricultural and Biological Science 6: 189-195.

Thakur D, Yadav A, Gogoi BK, Bora TC (2007) Isolation and screening of Streptomyces in soil of protected forest areas from the states of Assam and Tripura, India, for antimicrobial metabolites. Journal de Mycologie Médicale/Journal of Medical Mycology 17: 242-249.

Van Dam P, de Sain M, ter Horst A, van der Gragt M, Rep M (2017) Comparative genomics-based markers: discrimination of host-specificity in Fusarium oxysporum. Applied and Environmental Microbiology 84: 1. Doi: 10.1128 / AEM.01868-17.

Xue C, Penton RC, Shen Z, Zhang R, Huang Q, Li R, et al. (2015) Manipulating the banana rhizosphere microbiome for biological control of Panama disease. Scientific Reports 5: 11124. Doi: 10.1038/srep11124.

Yanet SL, Cabrales CP (2016) Identificación de especies de cepas nativas de Trichoderma sp. y Bacillus sp. y evaluación de su potencial antagonista in vitro frente al hongo fitopatógeno nativo Moniliophthora roreri en el departamento de Norte de Santander. Respuestas 13: 45-56.

Zhao B, Yan J, Zhang S, Liu X, Gao Z (2014) Phylogeny and pathogenicity of Fusarium spp. isolated from greenhouse melon soil in Liaoning Province. Saudi Journal of Biological Sciences 21: 374-379. 
